\title{
Um Estudo Empírico utilizando o Índice de Oportunidade Humana para o Brasil
}

\section{An Empirical Study using Human Opportunity Index for Brazil}

\author{
Daniel Suliano* \\ Jaime Jesus Filho**
}

\begin{abstract}
Resumo: O desenvolvimento das mais diversas habilidades de um indivíduo pode muitas vezes estar associado à desigualdade de acesso a um conjunto de oportunidades durante a infância, tanto em razão das escolhas sociais da sociedade à qual ele pertence, como também de circunstâncias que estejam fora de seu controle ao nascer. Nesses termos, e objetivando a projeção de políticas públicas de longo prazo, foi desenvolvido o Índice de Oportunidade Humana ( $\mathrm{IOH}$ ), indicador síntese da cobertura de bens e serviços básicos para o bem-estar de crianças a partir de parâmetros pré-determinados. Utilizando dados da Pesquisa Nacional por Amostra de Domicílios (PNAD) do IBGE para o Brasil nos anos de 2001-2011, foram elencados oito indicadores de serviços básicos, sendo cinco de dimensão habitacional e três de dimensão educacional, a partir de sete variáveis de circunstâncias. Os resultados mostram, além de uma heterogeneidade das oportunidades nas taxas de cobertura, uma ampliação da rede de acesso, não obstante diferenças entre os grupos de distintas circunstâncias.
\end{abstract}

Palavras-chave: Índice de oportunidade humana. Desigualdade de oportunidades. Circunstâncias.

\begin{abstract}
Development of the various skills of an individual can often is associated with unequal access to a set of opportunities during childhood as a result of social choices in addition to circumstances beyond their control to born. In these terms, and aiming to project long-term public policies, I developed the Human Opportunity Index (HOI), summary indicator of the coverage of basic goods and services of inequalities of opportunities for children with having parameters predetermined circumstances. The data used are from the National Household Sample Survey (PNAD) of IBGE for Brazil in the years 2001-2011. Were listed eight indicators of basic services being five housing dimension and three educational from seven variables circumstances. The results show diversity the opportunities besides in an expansion rates of coverage of the network access, notwithstanding differences between the groups in different circumstances.
\end{abstract}

Keywords: Human Opportunity Index; Inequality of Opportunities; Circumstances.

JEL Classification: D04; D31; D71.

\footnotetext{
* Doutor em Economia pelo Programa de Pós-gradação em Economia (CAEN) da Universidade Federal do Ceará (UFC). Analista de Políticas Públicas do Instituto de Pesquisa e Estratégia Econômica do Ceará (IPECE). E-mail: daniel.suliano@ipece.ce.gov.br

** PhD em Economia pela Universidade de Chicago. Consultor do Banco Mundial. E-mail: jjaimefilho@gmail.com
} 


\section{Introdução}

Em uma disputa econômica, os resultados ideais acontecem não quando todos terminam juntos a corrida, e sim quando partem de um mesmo ponto. Tal concepção de modelo social começou a se consolidar nos últimos três séculos, fruto das sociedades modernas, a partir das mudanças de paradigmas catapultadas por Hobbes (2009) [(1651)] e Locke (2001) [(1689)], nas quais a gênese do ente Estado estaria condicionada a sua capacidade de prover instrumentos de interesse geral para a sociedade. Até então, predominavam sociedades hierárquicas de natureza aristocrática, nas quais o progresso e as oportunidades de sucesso vinham predominantemente de laços de sangue. ${ }^{1}$

Mais recentemente, as escolas de filosofia política abordaram de forma distinta a questão da justiça distributiva. Rawls (1971), por exemplo, defende que as desigualdades econômicas e sociais devam ser permitidas caso beneficiem os menos favorecidos, tendo em vista que até os sistemas meritocráticos são moralmente contingentes, na medida em que favorecem aqueles de maior talento natural. Por outro lado, Nozick (1974), principal expoente libertário, argumenta que a ampliação de poderes por parte do Estado fere os direitos dos indivíduos e, portanto, sua liberdade individual.

Em termos econômicos, o conceito de justiça de acordo com Roemer (1998) passa a ser entendido em termos de igualdade de oportunidades, separando efeitos de circunstâncias, os quais estão fora do controle individual (exógenas a ele), e esforço, resultante das escolhas de cada um. Nesse contexto, a justiça no âmbito de políticas sociais, que formam a base para a construção do Índice de Oportunidade Humana $(\mathrm{IOH})$, ressalta como as circunstâncias, sejam elas originadas pelo gênero, escolaridade dos progenitores, renda, tamanho da família ou área de residência, podem vir a ser determinantes nos resultados originários dos esforços.

No Brasil, a discussão econômica do processo gerador de desigualdade dar-se-á a partir dos estudos seminais de Fishlow (1972) e Langoni (2005). ${ }^{2}$ O diagnóstico, à época, encontrou evidências da importância da educação na dinâmica do mercado de trabalho, como principal mecanismo influenciador na distribuição dos rendimentos. ${ }^{3}$

1 De acordo com Botton (2004), nestas sociedades a identidade de cada um dependia da origem de nascimento. Nas sociedades modernas, em sua maior parte, predomina o talento e/ou trabalho, tornando a posição social de cada um dependente de sua realização individual consubstanciada em sucesso financeiro.

2 Deve-se destacar que Furtado (1968) já discorre sobre a tendência de concentração de renda brasileira, ocorrida a partir do processo de industrialização e reforçada pela estrutura econômica nacional.

3 Posteriormente, diversas análises empíricas identificaram o lento progresso educacional como a variável que mais explica o alto grau de desigualdade de renda no Brasil (REIS; BARROS, 1990, 1991; LEAL; WERLANG, 1991; LAM; LEVINSON, 1992; LAM; SCHOENI, 1993; MENEZES- 
Ferreira (2000) discorre a respeito do mecanismo pelo qual essa desigualdade de renda permanece tão elevada no Brasil. A hipótese é que o país encontra-se num equilíbrio Pareto-inferior de um sistema dinâmico, em que três distribuições são determinadas simultaneamente, a saber: de educação, de riqueza e de poder político. Tal equilíbrio caracteriza-se por um círculo vicioso, no qual uma grande heterogeneidade educacional acarreta uma grande desigualdade de riqueza, que se transforma em grandes diferenças de poder político, que, por sua vez, geram uma política educacional que perpetua a desigualdade inicial.

Além disso, os mecanismos de transmissão da elevada desigualdade de renda via canal educacional podem também ser corroborados através da relação entre o histórico do indivíduo e a desigualdade de oportunidades entre diferentes grupos. Em que pese esses aspectos, a baixa mobilidade educacional no Brasil acaba sendo persistente no tempo por conta das diferenças de capital humano das famílias (FERREIRA; VELOSO, 2003; REIS; RAMOS, 2011).

Por outro lado, a década de 2000 foi caracterizada por uma queda contínua e robusta no grau de desigualdade, jogando luz sob quais mecanismos poderiam impulsionar maiores condições de equidade na sociedade brasileira. Uma vasta literatura produzida recentemente diagnosticou quais fatores estão por trás de uma das menores desigualdades nos últimos 40 anos, ${ }^{4}$ desde o surgimento da coleta de base de dados sistemática em pesquisas domiciliares.

Nos estudos de World Bank (2006), Barros et al. (2008), Barros et al. (2009b), Barros, Vega e Saavedra (2010) e Molinas et al. (2010), foi proposta a construção do Índice de Oportunidade Humana (IOH) a partir de circunstâncias exógenas que influenciam o acesso das crianças a bens e serviços básicos, necessários ao seu bem-estar. 5 Portanto, o IOH é uma métrica que combina oportunidades de determinados bens ou serviços, bem como o grau de desigualdade da distribuição de sua cobertura.

Nesse contexto, foi assim analisada a evolução do referido índice, a partir das sete circunstâncias elencadas em Barros et al. (2008), Barros et al. (2009b) e Molinas et al. (2010), e um compêndio de oito oportunidades nas dimensões de habitação/infraestrutura e educação ao longo da década de 2000. Além dessa breve introdução e das considerações finais, o presente trabalho apresenta mais duas seções. Na seção seguinte é discutida uma literatura do histórico da desigualdade

FILHO et al., 2000; MENEZES-FILHO, 2001). De acordo com Barros e Mendonça (1996), mesmo após considerar diferenças de experiência, gênero, raça, setor de atividade e região, a educação explica entre $30 \%$ a $50 \%$ da desigualdade total na renda brasileira.

4 Ver, por exemplo, Soares (2006), Hoffmann (2005), Barros et al. (2004, 2006a, 2006b, 2007a, 2007b, 2009a), Instituto de Pesquisa Econômica Aplicada (2006), Ferreira et al. (2006), Barros, Franco e Mendonça (2007a, 2007b, 2007c), Ramos (2007), Soares et al. (2007).

Em trabalho recente, Dill e Gonçalves (2012) replicam a análise de Barros et al. (2009b) e Molinas et al. (2010), estimando o IOH para o Brasil para os anos de 1999 e 2009 e uma decomposição do valor de Shapley para o índice de desigualdade de circunstâncias. 
brasileira, seguida de uma seção dividida em subseções, com a primeira contendo a definição e a descrição metodológica do $\mathrm{IOH}$, além de outra que discute a base de dados a ser analisada; por fim, uma subseção contém a análise e os comentários dos resultados produzidos.

\section{Desigualdade de Oportunidade no Brasil em Análise: uma Visão Geral}

A produção dos grandes latifúndios e a mão de obra escrava são apenas alguns dos traços marcantes do período colonial que foram determinantes no processo de sedimentação da desigualdade brasileira ao longo dos séculos. Essa naturalização ou institucionalização da desigualdade, que parece ter atravessado toda a história nacional, tem sido respaldada por uma literatura empírica recente. Dados do Censo do Império, de 1872, complementados por informações sobre rendimentos médios obtidos de registros de votantes da época, reproduzem um coeficiente de Gini no valor de 0,55 para aquele ano (VILLELA, 2013).

Destaca-se também, ao longo do século XIX, que, enquanto na Inglaterra e Estados Unidos emergia a noção de igualdade de direitos com busca de oportunidades equânimes para todos os indivíduos, a sociedade brasileira ainda mantinha a escravidão como estrutura central da base econômica, na qual a pobreza e a miséria humanas eram vistas como causas naturais e inevitáveis, sem a existência de um mecanismo institucional mínimo de amparo aos pobres (SCHWARTZMAN, 2004). De fato, em quase 200 anos de Independência, o Brasil não construiu um verdadeiro projeto nacional em termos de ganhos de bem-estar, que pudesse vir a beneficiar todos os seus cidadãos.

A partir do surgimento de microdados e, portanto, de análises mais precisas desse processo, grande parte do debate dentro da seara econômica, na tentativa de entender a iniquidade distributiva do Brasil se deu a partir dos trabalhos de Castro (1970), Fishlow (1972) e Langoni (2005), com ênfase na esfera educacional, mediante seu retorno e processo distributivo.

Nessa mesma perspectiva, Leal e Werlang (1991) seguem essa tônica, ao procurarem mostrar a relação entre distribuição de renda e nível de educação, entre meados dos anos de 1970 até meados dos anos de 1980. Eles observaram um aumento dos retornos pessoais por nível de escolaridade como responsável pelo aumento da desigualdade. Além disso, foi ainda observado que o arranjo institucional do ensino superior público brasileiro, por meio da gratuidade, acaba por favorecer poucos, potencializando ainda mais a desigualdade, tendo o agravante de o acesso ser obtido principalmente por indivíduos cujas famílias estão em condições mais favoráveis, o que lhes permite se prepararem em melhores escolas e terem boas classificações nos vestibulares. 
Adicionalmente, a elevada desigualdade de renda tende a tornar-se disfuncional, na medida em que causa descontrole social. De fato, muitas das mazelas da sociedade transfiguram-se em variáveis de cunho demográfico, tais como crianças nascidas em lares monoparentais, percentual de nascimentos de mães adolescentes e aumento da taxa de natalidade, de acordo com Hartung e Pessôa (2007). Para Barbosa Filho e Pessôa (2009), a opção brasileira no pós-guerra de não universalizar a educação fundamental no momento de transição demográfica, legou-nos, além de uma baixa produtividade do trabalho, a favelização das grandes cidades e explosão da criminalidade em áreas urbanas.

Destaca-se também que, mesmo em cenários favoráveis de baixa desigualdade, a fatia da renda total apropriada pela parcela 1\% mais rica da população é da mesma magnitude que a apropriada pelos 50\% mais pobres. Em outros termos, os $10 \%$ mais ricos se apropriam de mais de $40 \%$ da renda, enquanto os $40 \%$ mais pobres se apropriam de menos de 10\% (BARROS et al., 2006a; 2006b).

Para Osório (2005), soluções viáveis para se reduzir a pobreza no Brasil envolvem necessariamente alguma forma de deixar os ricos menos ricos. Com efeito, as evidências mostram não existir diferença na proporção média de pessoas em idade ativa, ou de ocupados, entre ricos e não ricos, que justifique as grandes desigualdades de rendimentos existentes. Além disso, uma suposta maior operosidade dos trabalhadores ricos não parece também ter relevância, uma vez que ambos os tipos de famílias estão empregando praticamente a plena capacidade seus trabalhadores. Ou seja, a composição, a organização e a divisão interna do trabalho das famílias ricas brasileiras não são suficientes para explicar porque elas se distinguem dos não ricos, em termos de renda per capita. É precisamente na remuneração desigual de cada grupo de trabalhadores em que se encontra a maior parte do diferencial entre ricos e não ricos (MEDEIROS, 2004).

Em uma extensa pesquisa, na tentativa de entender o progresso econômico das nações, Acemoglu e Robinson (2012) demonstram que cada sociedade funciona com um conjunto de regras políticas que são criadas e aplicadas pelo Estado e por cidadãos comuns. No entanto, em algumas sociedades, um círculo vicioso é instaurado e os detentores do poder econômico e político encontram meios de estruturar as instituições, de modo a assegurar a continuidade e a permanência das mesmas elites no poder.

No caso do Brasil, mesmo havendo legitimidade por parte das instituições, que garante o direito e a igualdade de condições a todos os cidadãos, os ricos brasileiros estão em ocupações que, além de receberem os maiores rendimentos, são também aquelas que se encontram em posições privilegiadas para influir no processo legislativo e judiciário e na máquina pública. Isso sem falar naqueles que ocupam postos nas elites políticas e que também pertencem às elites econômicas. Além disso, estão inseridos em redes privilegiadas e de posse de capital cultural, 
características que os ricos têm por terem nascido ricos e por terem convivido com outros ricos (MEDEIROS, 2005; OSÓRIO, 2005).

\section{A Construção do Índice de Oportunidade Humana ${ }^{6}$}

Uma sociedade que busca condições de oportunidades iguais no acesso a bens e serviços de suas crianças espera que, em um ambiente ideal, os anseios e as conquistas de cada um sejam legitimamente aceitáveis, caso reflitam diferenças de escolha, esforços ou talento individuais e não sejam resultado de circunstâncias pré-determinadas.

Com base nessa ideia, o Índice de Oportunidade Humana ( $\mathrm{IOH}$ ) tem como um de seus objetivos projetar políticas públicas de longo prazo e romper com a persistência dos ciclos intergeracionais de desigualdade aos moldes da função de bem-estar de Sen (1976), com foco nas crianças com idade entre zero e 16 anos (ver, por exemplo, WORLD BANK, 2006; BARROS et al., 2008; BARROS et al., 2009b; BARROS; VEJA; SAAVEDRA, 2010; MOLINAS et al.. 2010).

De forma analítica, estimar a cobertura e a desigualdade de oportunidades requer elencar variáveis determinantes para o desenvolvimento do indivíduo que estejam, ao mesmo tempo, fora de seu controle (exógenas para os cidadãos) e que possam sofrer influências de escolhas (endógenas para a sociedade). Em Barros et al. (2009b) e Molinas et al. (2010), por exemplo, foram considerados cinco indicadores de bens e serviços considerados básicos, sendo dois de dimensão educacional e três de habitação/infraestrutura.

O foco nas crianças, com base nos parâmetros de cobertura em bens e serviços básicos e desigualdades a eles associados, leva o $\mathrm{IOH}$ a procurar "nivelar o campo de jogo". Além disso, insere-se no centro do debate político, na medida em que intervenções precoces ao longo do ciclo de vida apresentam maior probabilidade de equalizar as condições de oportunidade, tornando, assim, as políticas mais eficazes do que aquelas feitas mais tardiamente, seja qual for o campo ideológico a qual pertence seu formulador (BARROS et al., 2009b).

$\mathrm{O} \mathrm{IOH}$, particularmente, é formado pela taxa de cobertura $C$ e um índice de desigualdade $D$. O índice $D$ é interpretado como o quantitativo de crianças que teria de ser realocado entre grupos de circunstâncias distintas vis-à-vis aqueles com acesso à mesma dimensão de oportunidades. Dessa forma, o $(1-D)$ corresponde ao percentual de oportunidades que foram igualmente distribuídas. Assim, o IOH pode ser assim representado:

$$
I O H=C \times(1-D)
$$

6 Para mais detalhes da dedução do $\mathrm{IOH}$, ver Apêndice. 
O IOH pode também ser interpretado como o acesso a uma oportunidade, em termos de taxa de cobertura descontada pela sua alocação de forma desigual. Sendo assim, pode-se mensurar a melhora de acesso do bem ou serviço básico de um determinado país por meio do $\mathrm{IOH}$, caso ocorra um aumento na cobertura média e/ou através de um aumento da igualdade de oportunidade existente desse acesso. $^{7}$

\subsection{Métrica para o Índice de Desigualdade (D) ${ }^{8}$}

A desigualdade de oportunidades (índice $D$ ) é uma medida relativa de acesso médio de um bem ou serviço (dimensão), para grupos definidos por características de circunstâncias, em comparação com a taxa média de acesso da mesma dimensão, em análise para a população em geral.

Para uma amostra aleatória de $i$ crianças em uma dada oportunidade $\left(I_{i}=1\right.$ se o grupo tem acesso e $I_{i}=0$ caso contrário) e um vetor de $m$ circunstâncias ${ }^{9}$ $x_{i}=\left(x_{1 i}, \ldots, x_{\dot{\boldsymbol{m}}}\right)$, pode-se escrever o índice de desigualdade de oportunidade alternativamente como:

$$
D=\frac{E|P(I=1 \mid x)-P(I=1)|}{2 P(I=1)}
$$

reescrevendo $P(I=1)=E(P(I=1 \mid x)$ tem-se:

$$
D=\frac{E \mid P(I=1 \mid x)-E(P(I=1 \mid x) \mid}{2 E(P(I=1 \mid x)}
$$

Na equação 3, a estimação de $D$ é feita com base em algum grupo definido, em termos de circunstâncias para uma determinada taxa de cobertura de uma oportunidade, expressa por $P(I=1 \mid x)$. As estimativas se dão por meio de regressão logística, tal que:

$$
\operatorname{Ln}\left(\frac{P\left(I=1 \mid x_{1}, \ldots, x_{m}\right)}{1-P\left(I=1 \mid x_{1}, \ldots, x_{m}\right)}\right)=\sum_{k=1}^{m} h_{k}\left(x_{k}\right)
$$

$7 \quad$ Resultados de Barros et al. (2009b) e Molinas et al. (2010) para dados da América Latina e Caribe, em um intervalo de 10 anos (1995-2005), mostram que dois terços das melhorias no IOH na região foram decorrentes do aumento no fornecimento de oportunidades disponíveis e o terceiro terço restante por uma redução da desigualdade no acesso.

8 Esta subseção é baseada em Barros, Vega e Saavedra (2010).

9 A subseção 3.3 descreve em maiores detalhes as dimensões de oportunidade e as circunstâncias a serem aqui analisadas (ver Quadros 1 e 2 na referida seção). 
com $x_{k}$ denotando um vetor de variáveis de $k$ circunstâncias tal que $x=\left(x_{1}, \ldots, x_{m}\right)$. As funções $\left\{h_{k}\right\}$ estão em consonância com as circunstâncias delineadas e $h_{k}\left(x_{k}\right)=x_{k} \beta_{k}$, sendo, portanto, lineares nos parâmetros. A probabilidade prevista de acesso a uma determinada oportunidade de uma criança $i$ poderá ser obtida por:

$$
\hat{p}_{i}=\frac{\exp \left(\hat{\beta}_{0}+\sum_{k=1}^{m} x_{k i} \hat{\beta}_{k}\right)}{1+\exp \left(\hat{\beta}_{0}+\sum_{k=1}^{m} x_{k i} \hat{\beta}_{k}\right)}
$$

e sendo a taxa média de acesso denotada por $\bar{p}$, e $w_{i}=\frac{1}{n}$ as ponderações, chega-se às seguintes expressões finais que denotam a estimativa de $D$ :

$$
\bar{p}=\sum_{i=1}^{n} w_{i} \hat{p}_{i} \text { e } \hat{D}=\frac{1}{2 p^{-}} \sum_{i=1}^{n} w_{i}\left|\hat{p}_{i}-\bar{p}\right|
$$

\subsection{Base de Dados: Conjunto de Circunstâncias e Dimensões de Oportunidade}

Para a construção dos indicadores, foi utilizada como base a Pesquisa Nacional por Amostras de Domicílios (PNAD) do Instituto Brasileiro de Geografia e Estatística (IBGE) nos anos de 2001 a $2011 .{ }^{10}$ Nesse período, o Brasil conseguiu conjugar crescimento econômico com redução na desigualdade (Gráfico 1), combinação não usual para o padrão de desenvolvimento brasileiro no pós-guerra. ${ }^{11}$

10 Exceto o ano de 2010, tendo ocorrido Censo Demográfico e não PNAD. Ver (IBGE, 2017).

11 Ver, por exemplo, Ferreira e Veloso (2013). 
Gráfico 1- Evolução da taxa de crescimento do produto e do índice de gini (renda domiciliar per capita).

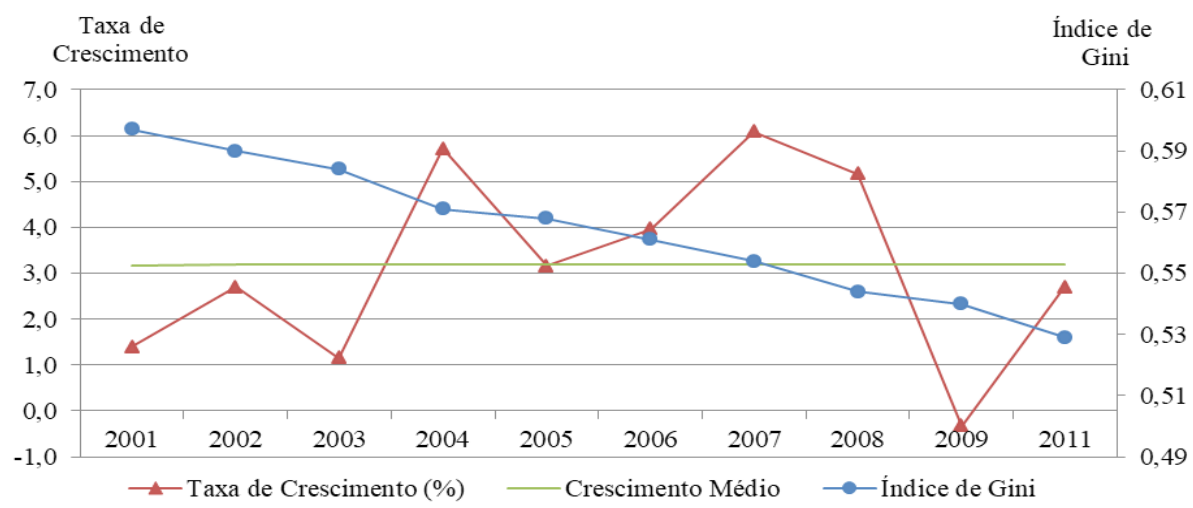

Fonte: Elaboração própria a partir de IBGE (2017).

Conforme visto nas subseções anteriores, determinar desigualdade de oportunidade a partir do $\mathrm{IOH}$ requer, além de circunstâncias exógenas ao nascimento, dimensões no que concerne ao acesso de bens e serviços considerados básicos ao bem-estar. Com relação às variáveis exógenas de circunstâncias, seguiu-se a linha de Barros et al. (2009b) e Molinas et al. (2010) descritas no Quadro 1.

Quadro 1- Descrição das circunstâncias para aferição do índice de desigualdade $D$.

\begin{tabular}{|l|l|}
\hline \multicolumn{1}{|c|}{$\begin{array}{c}\text { Dimensão da } \\
\text { Circunstância }\end{array}$} & \multicolumn{1}{c|}{ Descrição } \\
\hline Gênero & Masculino/Feminino. \\
\hline $\begin{array}{l}\text { Escolaridade da pessoa } \\
\text { de referência }\end{array}$ & $\begin{array}{l}\text { Pessoa responsável pela unidade domiciliar (ou } \\
\text { pela família) ou que assim fosse considerada pelos } \\
\text { demais membros. A classificação segundo os anos } \\
\text { de estudo foi obtida em função da série e do nível } \\
\text { ou grau que a pessoa estava frequentando ou havia } \\
\text { frequentado, considerando a última série concluída } \\
\text { com aprovação. }\end{array}$ \\
\hline $\begin{array}{l}\text { Número de crianças no } \\
\text { domicílio }\end{array}$ & $\begin{array}{l}\text { Total de crianças de 0 a 16 anos de idade residentes } \\
\text { no mesmo domicílio. }\end{array}$ \\
\hline Renda familiar per capita & $\begin{array}{l}\text { Razão entre o total de rendimentos de todas as pessoas } \\
\text { da família e o seu número de componentes. }\end{array}$ \\
\hline $\begin{array}{l}\text { Família com formação } \\
\text { uniparental }\end{array}$ & $\begin{array}{l}\text { Domicílio onde reside apenas um progenitor (pai ou } \\
\text { mãe solteiro). }\end{array}$ \\
\hline Área de residência & $\begin{array}{l}\text { Código de situação censitária: área urbana ou área } \\
\text { rural }\end{array}$ \\
\hline
\end{tabular}

Fonte: Elaboração própria. 
Além disso, foram elencadas oito dimensões de oportunidades para bens e serviços básicos sendo cinco delas de dimensão habitacional/infraestrutura - acesso à água, saneamento, eletricidade, telefonia celular e internet - e mais três de dimensão educacional - início do ensino fundamental na idade certa, frequência à pré-escola, para aqueles de idade de 4 e 5 anos, e frequência à escola, para aqueles com idade de 10 a 14 anos. No Quadro 2 são descritas em maiores detalhes as oportunidades elencadas.

As dimensões de acesso à agua e saneamento são indicadoras de saúde pública. Saneamento adequado e melhoria da higiene, bem como água potável, apresentam tendência na redução das taxas de mortalidade infantil em países em desenvolvimento, de acordo Rutstein (2000). Seus efeitos também se transfiguram em reduzida incidência de diarreia e outros efeitos a longo prazo, tais como desnutrição, infecções oportunistas (pneumonia, por exemplo) e nanismo físico e metal (MOLINAS et al., 2010).

Para a dimensão de acesso à eletricidade, sua capilaridade é ampla, variando desde melhorias nas condições de saúde à externalidades nas dimensões educacionais. De fato, o uso de fogões elétricos em substituição aos de queima de biomassa reduz os riscos de infecções respiratórias, a maior causa da mortalidade infantil em países em desenvolvimento (ARBEX et al., 2004). Adicionalmente, Kaufman et al. (2000) ressaltam a redução dos riscos de envenenamento e melhorias na saúde das famílias da área rural ao ser feito uso da energia solar, em substituição à queima de querosene.

Deve-se ressaltar que, nos estudos seminais de Barros et al. (2009b) e Molinas et al. (2010), estão presentes nas dimensões de habitação o acesso à água, saneamento e eletricidade, enquanto nas dimensões de educação, foram utilizados como parâmetros a conclusão da sexta série na idade certa e a frequência escolar de crianças na idade de 10 a 14 anos. Em Barros et al. (2009b), é destacado que outras versões do índice podem ampliar o conjunto de oportunidades, aumentando também o horizonte de análise, com propostas mais eficazes de políticas públicas.

Em vista disso, a PNAD permite a ampliação do espectro de análise, estendendo o conjunto de oportunidades, tanto no campo da infraestrutura como no campo educacional. Nas dimensões de educação, além da frequência escolar na idade de 10 a 14 anos, foi incluída a frequência escolar à pré-escola para a idade de 4 e 5 anos e a inicialização do ensino fundamental na idade certa. A inclusão de duas dimensões educacionais parte dos avanços ocorridos na quase universalização do ensino fundamental no país, na década de 1990, principalmente com a ampliação dos recursos instituídos pelo Fundef, reduzindo a desigualdade do gasto por aluno e aumentando a eficiência de alocação de recursos (VELOSO, 2009).

No que concerne as dimensões de infraestrutura (acesso à telefonia celular e acesso à internet), Barros et al. (2009b) destacam seus efeitos simbióticos com a 
dimensão acesso à eletricidade. Neste caso, a dimensão de eletricidade, ao melhorar as condições de estudo noturnas, pode potencializar essas dimensões na medida em que amplia o acesso à informação e libera tempo dos pais de tarefas domésticas, podendo, assim, alocá-lo na criação dos filhos. Ademais, essas ferramentas de difusão tecnológica geram spilovers e permitem acesso a outras ferramentas, como atestam Cristia et al. (2012) no uso de laptop em áreas rurais do Peru, impactando em maior desempenho cognitivo e testes de influência verbal e matemática.

Quadro 2 - Descrição das condições de oportunidades para o índice de oportunidade humana

\begin{tabular}{|c|c|}
\hline $\begin{array}{c}\begin{array}{c}\text { Dimensão das condições } \\
\text { de oportunidade }\end{array} \\
\end{array}$ & Descrição \\
\hline \multicolumn{2}{|c|}{ Dimensões de Habitação/Infraestrutura } \\
\hline Acesso à água & $\begin{array}{l}\text { Existência de água canalizada nos domicílios } \\
\text { particulares permanentes. }\end{array}$ \\
\hline Acesso a saneamento & $\begin{array}{l}\text { Existência de banheiro ou sanitário, para uso de } \\
\text { moradores, no domicílio particular permanente, } \\
\text { no terreno ou na propriedade em que estava } \\
\text { situado. }\end{array}$ \\
\hline Acesso à eletricidade & $\begin{array}{l}\text { A forma de iluminação elétrica utilizada no } \\
\text { domicílio particular permanente foi assim } \\
\text { classificada quando o domicílio tivesse } \\
\text { iluminação elétrica proveniente de rede geral, } \\
\text { conversor de energia solar, entre outros. }\end{array}$ \\
\hline Acesso à telefonia celular & $\begin{array}{l}\text { Acesso de algum morador do domicílio } \\
\text { particular à linha telefônica móvel (telefone } \\
\text { móvel celular). }\end{array}$ \\
\hline Acesso à internet & $\begin{array}{l}\text { Nos domicílios particulares em que havia } \\
\text { microcomputador foi pesquisado se era utilizado } \\
\text { acesso à internet. }\end{array}$ \\
\hline \multicolumn{2}{|r|}{ Dimensões de Educação } \\
\hline $\begin{array}{l}\text { Início do ensino fundamental } \\
\text { na idade certa }\end{array}$ & \begin{tabular}{|lllll} 
Entrada na idade correta & no Ensino \\
Fundamental." & & & \\
\end{tabular} \\
\hline $\begin{array}{l}\text { Frequência à pré-escola na } \\
\text { idade de } 4 \text { e } 5 \text { anos }\end{array}$ & $\begin{array}{l}\text { Crianças com idade de } 4 \text { e } 5 \text { anos que estejam } \\
\text { frequentando escola ou creche, independente } \\
\text { se estejam no período letivo correspondente a } \\
\text { sua idade escolar. }\end{array}$ \\
\hline $\begin{array}{l}\text { Frequência à escola na idade } \\
\text { de } 10 \text { a } 14 \text { anos }\end{array}$ & $\begin{array}{l}\text { Crianças na faixa etária de } 10 \text { a } 14 \text { anos que } \\
\text { estejam frequentando a escola, independente se } \\
\text { estejam no período letivo correspondente a sua } \\
\text { idade escolar. }\end{array}$ \\
\hline
\end{tabular}

Fonte: Elaboração própria.

Nota:" A partir de 2007, a entrada no Ensino Fundamental passou a ser aos 6 anos (no regime de 9 anos). Até 2006, a entrada era aos 7 anos (no regime de 8 anos). 


\subsection{Resultados e Discussões}

Nas Tabelas 1, 2 e 3 são apresentados, respectivamente, os resultados da taxa de cobertura (C), do índice de desigualdade (D) e do Índice de Oportunidade Humana $(\mathrm{IOH})$. Para os três indicadores foi calculada a série do período em análise para cada uma das oito oportunidades elencadas. A taxa de cobertura tem uma interpretação intuitiva, tendo em conta que quanto mais próximo de $100 \%$, maior a sua amplitude em termos de acesso.

Tabela 1- Evolução da Taxa de Cobertura (C), em \%

\begin{tabular}{|c|c|c|c|c|c|c|c|c|c|c|}
\hline Dimensões de oportunidades & 2001 & 2002 & 2003 & 2004 & 2005 & 2006 & 2007 & 2008 & 2009 & 2011 \\
\hline \multicolumn{11}{|l|}{ Habitação/Infraestrutura } \\
\hline Água & $81,1 \%$ & $83,4 \%$ & $84,0 \%$ & $83,7 \%$ & $84,6 \%$ & $85,9 \%$ & $87,5 \%$ & $88,6 \%$ & $89,4 \%$ & $90,7 \%$ \\
\hline Saneamento & $57,9 \%$ & $59,6 \%$ & $60,5 \%$ & $60,2 \%$ & $60,9 \%$ & $62,1 \%$ & $65,8 \%$ & $65,7 \%$ & $65,2 \%$ & $70,3 \%$ \\
\hline Eletricidade & $94,0 \%$ & $95,2 \%$ & $95,5 \%$ & $95,1 \%$ & $95,4 \%$ & $96,2 \%$ & $97,3 \%$ & $97,9 \%$ & $98,4 \%$ & $99,0 \%$ \\
\hline Acesso a telefone celular & $25,8 \%$ & $29,2 \%$ & $33,2 \%$ & $43,2 \%$ & $56,2 \%$ & $61,5 \%$ & $66,4 \%$ & $76,2 \%$ & $79,8 \%$ & $88,9 \%$ \\
\hline Acesso à internet & $5,9 \%$ & $7,3 \%$ & $8,0 \%$ & $8,5 \%$ & $9,8 \%$ & $12,2 \%$ & $15,4 \%$ & $19,3 \%$ & $44,6 \%$ & $33,1 \%$ \\
\hline \multicolumn{11}{|l|}{ Educação } \\
\hline $\begin{array}{l}\text { Início do Ensino Fundamental } \\
\text { na idade certa }\end{array}$ & $19,0 \%$ & $19,9 \%$ & $20,2 \%$ & $21,3 \%$ & $21,4 \%$ & $21,8 \%$ & $21,1 \%$ & $20,2 \%$ & $20,3 \%$ & $22,3 \%$ \\
\hline $\begin{array}{l}\text { Frequenta a pré-escola, idade } \\
4-5 \text { anos }\end{array}$ & $54,9 \%$ & $56,6 \%$ & $59,0 \%$ & $61,5 \%$ & $62,8 \%$ & $67,7 \%$ & $70,1 \%$ & $72,9 \%$ & $75,0 \%$ & $77,4 \%$ \\
\hline $\begin{array}{l}\text { Frequenta a escola, idade } 10 \text { - } \\
14 \text { anos }\end{array}$ & $96,2 \%$ & $96,8 \%$ & $97,1 \%$ & $96,8 \%$ & $97,1 \%$ & $97,3 \%$ & $97,3 \%$ & $97,7 \%$ & $97,7 \%$ & $98,3 \%$ \\
\hline
\end{tabular}

Fonte: Elaboração própria a partir de IBGE (2017).

Nota: Harmonizados pelo CEDLAS e Banco Mundial.

Pode-se observar uma heterogeneidade no acesso aos bens e serviços. Além disso, exceto para as coberturas "eletricidade" e "início do ensino fundamental na idade certa", as demais apresentaram uma tendência de crescimento vertiginosa. No caso da primeira, a baixa taxa de ampliação deve-se, principalmente, à quase universalização do serviço; enquanto no caso da segunda, seu crescimento ao longo do período se expande lentamente a $1 \%$ a.a., tendo a menor cobertura em 2011 entre todas as dimensões analisadas. Já a dimensão "frequência à pré-escola para idade 10-14 anos", mesmo com uma taxa de expansão lenta, detém uma taxa de cobertura quase universalizada ao final da série. 
No quesito "água", a expansão acontece também a taxas lentas, mas partindo de uma base alta (81\%), finalizando a série com pouco menos de 91\%. Por sua vez, a cobertura de "acesso a telefone celular" e "acesso à internet" demonstram uma boa perspectiva, refletindo uma melhor eficiência dos serviços ao longo da década de $2000 .{ }^{12}$

No aspecto do serviço de "saneamento", deve-se destacar que, apesar de em 2001 a cobertura restringir-se a 58\%, em 2011 há uma ampliação para pouco mais de $70 \%$ do total. Uma tendência também importante a ser considerada é a "frequência à pré-escola para idade 4-5 anos", reflexo possivelmente da ampliação da rede de creches e maior taxa de participação das mulheres no mercado de trabalho. Em 2011, a taxa de cobertura para essa oportunidade já chegara a pouco mais de $77 \%$.

Como descrito acima, a alocação das oportunidades é ponderada pelo índice de desigualdade $D$, conforme a construção do $\mathrm{IOH}$. Em que pese a diferença em termos da dinâmica de acesso, os Gráficos 2 e 3 separam as dimensões "acesso a telefone celular" e "acesso à internet" das demais.

Gráfico 2- Evolução do índice de desigualdade (D) - dimensões selecionadas

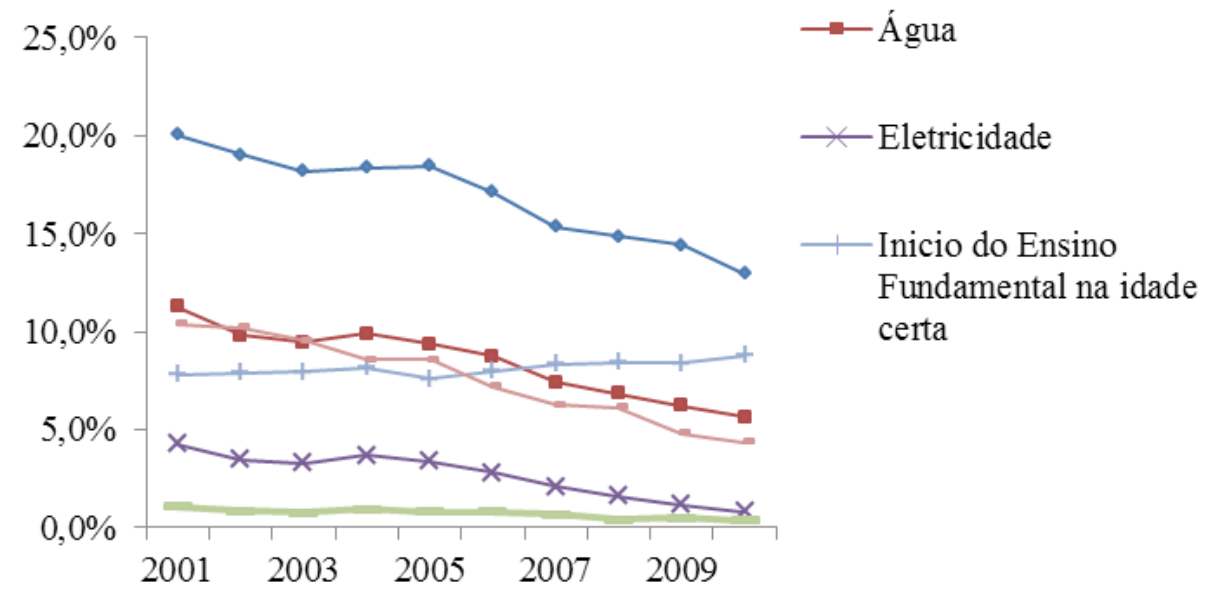

Fonte: Elaboração própria a partir de IBGE (2017).

Nota: Harmonizados pelo CEDLAS e Banco Mundial.

12 A privatização do setor de telecomunicações no final da década de 1990 ocasionou uma maior oferta de serviços, além do aumento da concorrência, o que, aliado ao aumento do poder aquisitivo no período posterior, veio permitir acesso mais amplo aos serviços. 
Gráfico 3- Evolução do índice de desigualdade (D) - dimensões selecionadas

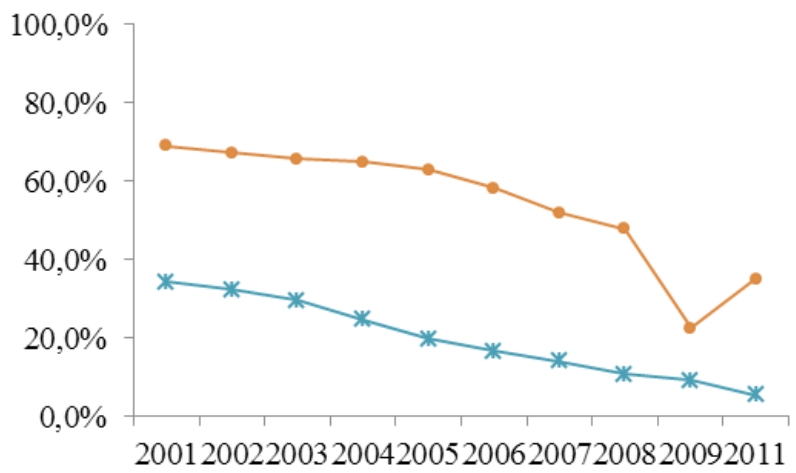

* Acesso a telefone celular

Fonte: Elaboração própria a partir de IBGE (2017).

Nota: Harmonizados pelo CEDLAS e Banco Mundial.

Na Tabela 2 encontram-se os resultados em maiores detalhes. Em certa medida, refletem a imagem inversa da tabela anterior, considerando que uma maior desigualdade deve ser entendida como uma realocação das oportunidades disponíveis daqueles grupos mais favorecidos aos grupos menos favorecidos, no intuito de gerar um maior alcance de igualdade de oportunidade.

De fato, cabe destacar o acesso às condições de "saneamento" e "início do ensino fundamental na idade certa". Em ambos os casos, apesar de as estimativas ao longo da série exibirem uma tendência de redução da desigualdade, ainda do ponto de vista relativo, as diferenças entre os grupos de distintas circunstâncias são prementes. Em 2011, por exemplo, 13\% das oportunidades no acesso ao saneamento precisariam ser realocadas ao grupo de pior situação, de modo a se alcançar uma maior igualdade de oportunidade.

Por sua vez, deve-se também ressaltar a evolução dos serviços de comunicação reportados no Gráfico 3, com destaque para a ainda persistente desigualdade na oportunidade "acesso à internet" para o último ano da série. No caso, 35\% delas precisariam ser realocadas, a fim de se atingir a igualdade de oportunidades.

Nas dimensões "eletricidade" e "frequência à escola para idade 10-14 anos" os resultados se dão de forma mais estanque, enquanto "água" e "frequência à pré-escola para idade 4-5 anos" apresentam clara redução da desigualdade ao longo da década. 
Tabela 2- Evolução do Índice de Desigualdade (D), em \%

\begin{tabular}{|c|c|c|c|c|c|c|c|c|c|c|}
\hline Dimensões de oportunidades & 2001 & 2002 & 2003 & 2004 & 2005 & 2006 & 2007 & 2008 & 2009 & 2011 \\
\hline \multicolumn{11}{|l|}{ Habitação/Infraestrutura } \\
\hline Água & $11,3 \%$ & $9,8 \%$ & $9,4 \%$ & $9,9 \%$ & $9,4 \%$ & $8,7 \%$ & $7,4 \%$ & $6,8 \%$ & $6,2 \%$ & $5,6 \%$ \\
\hline Saneamento & $20,0 \%$ & $19,0 \%$ & $18,2 \%$ & $18,4 \%$ & $18,4 \%$ & $17,1 \%$ & $15,4 \%$ & $14,9 \%$ & $14,4 \%$ & $12,9 \%$ \\
\hline Eletricidade & $4,3 \%$ & $3,5 \%$ & $3,3 \%$ & $3,7 \%$ & $3,4 \%$ & $2,8 \%$ & $2,1 \%$ & $1,6 \%$ & $1,2 \%$ & $0,8 \%$ \\
\hline Acesso a telefone celular & $34,3 \%$ & $32,3 \%$ & $29,6 \%$ & $24,7 \%$ & $19,7 \%$ & $16,7 \%$ & $14,1 \%$ & $10,7 \%$ & $9,1 \%$ & $5,5 \%$ \\
\hline Acesso à internet & $69,0 \%$ & $67,3 \%$ & $65,6 \%$ & $64,8 \%$ & $62,9 \%$ & $58,2 \%$ & $52,1 \%$ & $47,8 \%$ & $22,5 \%$ & $35,0 \%$ \\
\hline \multicolumn{11}{|l|}{ Educação } \\
\hline $\begin{array}{l}\text { Início do Ensino Fundamental } \\
\text { na idade certa }\end{array}$ & $7,8 \%$ & $7,9 \%$ & $7,9 \%$ & $8,2 \%$ & $7,6 \%$ & $8,0 \%$ & $8,3 \%$ & $8,5 \%$ & $8,4 \%$ & $8,8 \%$ \\
\hline $\begin{array}{l}\text { Frequenta a pré-escola, idade } \\
4-5 \text { anos }\end{array}$ & $10,4 \%$ & $10,2 \%$ & $9,6 \%$ & $8,6 \%$ & $8,6 \%$ & $7,2 \%$ & $6,2 \%$ & $6,1 \%$ & $4,8 \%$ & $4,3 \%$ \\
\hline $\begin{array}{l}\text { Frequenta a escola, idade } 10 \text { - } \\
14 \text { anos }\end{array}$ & $1,1 \%$ & $0,9 \%$ & $0,8 \%$ & $0,9 \%$ & $0,8 \%$ & $0,8 \%$ & $0,7 \%$ & $0,4 \%$ & $0,5 \%$ & $0,4 \%$ \\
\hline
\end{tabular}

Fonte: Elaboração própria a partir de IBGE (2017).

Nota: Harmonizados pelo CEDLAS e Banco Mundial.

Finalmente, a Tabela 3 reporta os resultados para o Índice de Oportunidade Humana ( $\mathrm{IOH})$. Semelhantemente aos resultados da taxa de cobertura, "eletricidade" e "frequência à escola para idade 10-14 anos" são dois indicadores com taxas de proporção do total de oportunidades disponíveis em 2001 já elevadas, o que leva, por conseguinte, a uma baixa tendência de crescimento e quase universalização em 2011. Por sua vez, a dimensão inicialização do ensino fundamental na idade correta apresenta condições de oportunidade ainda em níveis modestos, dada a sua lenta taxa de expansão no período.

Cabe mais uma vez mencionar o vertiginoso crescimento do "acesso à telefonia celular" e "acesso à internet", assim como a tendência à universalização da "água". No entanto, tanto "saneamento" como "frequência à pré-escola para idade 4-5 anos" ainda estão bem aquém dos demais indicadores, particularmente esta última. O caso do acesso ao saneamento preocupa em razão de seus desdobramentos na formação na qualidade do capital humano, conforme discutido anteriormente. No ano de 2011 , apenas $61 \%$ das oportunidades de saneamento estavam igualmente distribuídas para as crianças de 0 a 16 anos no Brasil. 
Tabela 3 - Evolução do índice de oportunidade humana (IOH), crianças de 016 anos, em \%

\begin{tabular}{|c|c|c|c|c|c|c|c|c|c|c|}
\hline Dimensões de oportunidades & 2001 & 2002 & 2003 & 2004 & 2005 & 2006 & 2007 & 2008 & 2009 & 2011 \\
\hline \multicolumn{11}{|l|}{ Habitação/Infraestrutura } \\
\hline Água & $71,9 \%$ & $75,2 \%$ & $76,1 \%$ & $75,4 \%$ & $76,6 \%$ & $78,4 \%$ & $81,0 \%$ & $82,5 \%$ & $83,8 \%$ & $85,6 \%$ \\
\hline Saneamento & $46,3 \%$ & $48,2 \%$ & $49,5 \%$ & $49,1 \%$ & $49,7 \%$ & $51,5 \%$ & $55,7 \%$ & $56,0 \%$ & $55,8 \%$ & $61,2 \%$ \\
\hline Eletricidade & $90,0 \%$ & $91,8 \%$ & $92,4 \%$ & $91,6 \%$ & $92,2 \%$ & $93,5 \%$ & $95,2 \%$ & $96,3 \%$ & $97,2 \%$ & $98,2 \%$ \\
\hline Acesso a telefone celular & $17,0 \%$ & $19,8 \%$ & $23,4 \%$ & $32,6 \%$ & $45,1 \%$ & $51,2 \%$ & $57,0 \%$ & $68,1 \%$ & $72,5 \%$ & $84,0 \%$ \\
\hline Acesso à internet & $1,8 \%$ & $2,4 \%$ & $2,7 \%$ & $3,0 \%$ & $3,6 \%$ & $5,1 \%$ & $7,4 \%$ & $10,1 \%$ & $12,5 \%$ & $21,5 \%$ \\
\hline \multicolumn{11}{|l|}{ Educação } \\
\hline $\begin{array}{l}\text { Início do Ensino Fundamental } \\
\text { na idade certa }\end{array}$ & $17,5 \%$ & $18,4 \%$ & $18,6 \%$ & $19,5 \%$ & $19,7 \%$ & $20,1 \%$ & $19,3 \%$ & $18,5 \%$ & $18,6 \%$ & $20,3 \%$ \\
\hline $\begin{array}{l}\text { Frequenta a pré-escola, idade } 4 \text { - } \\
5 \text { anos }\end{array}$ & $49,2 \%$ & $50,8 \%$ & $53,4 \%$ & $56,2 \%$ & $57,4 \%$ & $62,8 \%$ & $65,7 \%$ & $68,4 \%$ & $71,4 \%$ & $74,1 \%$ \\
\hline $\begin{array}{l}\text { Frequenta a escola, idade 10-14 } \\
\text { anos }\end{array}$ & $95,2 \%$ & $96,0 \%$ & $96,4 \%$ & $95,9 \%$ & $96,3 \%$ & $96,5 \%$ & $96,7 \%$ & $97,3 \%$ & $97,2 \%$ & $98,0 \%$ \\
\hline
\end{tabular}

Fonte: Elaboração própria a partir de IBGE (2017).

Nota: Harmonizados pelo CEDLAS e Banco Mundial.

\section{Considerações Finais}

A persistente e contínua desigualdade brasileira é resultado de um processo histórico, que foi endossado por um modelo em que as condições de acesso às oportunidades básicas dos indivíduos estiveram condicionadas por mecanismos nos quais as condições ideais de uma disputa econômica justa não se deram de modo que todos começassem do mesmo ponto de partida.

O Índice de Oportunidade Humana ( $\mathrm{IOH})$, com o objetivo de projetar políticas públicas de longo prazo, é uma medida de desigualdade de oportunidades que busca mensurar o acesso à cobertura de bens e serviços básicos para o bem-estar das crianças a partir de fatores pré-determinados. Assim, no seu cômputo, o IOH elenca variáveis de circunstâncias que estejam fora do controle das crianças - exógenas para os cidadãos -, além daquelas que podem vir a ser alteradas por escolhas sociais - endógenas para a sociedade.

Neste trabalho, foram utilizados dados da Pesquisa Nacional por Amostra de Domicílios (PNAD) do IBGE para o Brasil nos anos de 2001 a 2011, período caracterizado por uma relativa estabilidade macroeconômica e melhorias nos indicadores sociais.

Dentro desse contexto, foram elencados oito indicadores de bens e serviços, sendo cinco de dimensão habitacional - acesso à agua, saneamento, eletricidade, telefonia celular e internet - e três de dimensão educacional - inicialização do 
ensino fundamental na idade certa, frequência escolar na idade de 4 e 5 anos, e frequência escolar na idade de 10 a 14 anos - a partir de sete variáveis de circunstâncias: gênero, raça, renda familiar per capita, escolaridade da pessoa de referência, presença de um dos pais ou ambos na moradia, número de crianças no domicilio com idade entre 0 e 16 anos e área urbana.

Os resultados para o $\mathrm{IOH}$ demonstram crescimento do "acesso à dimensão telefonia celular e internet" bem como uma tendência à universalização da oportunidade acesso à "água"; não obstante a ocorrência ainda adversa de oportunidades das dimensões de "saneamento" e "frequência à pré-escola para crianças com idade entre 4 e 5 anos". As dimensões de "eletricidade" e "frequência à escola para idade 10-14 anos" estão praticamente universalizadas. Deve-se também destacar que a "inicialização do ensino fundamental na idade correta", além de apresentar condições de oportunidade em níveis baixos, teve ainda uma lenta taxa de expansão no período em análise.

Nesse contexto, são grandes os desafios a serem alcançados em termos de políticas públicas. De acordo com as evidências, a prevenção, segundo as recomendações fitossanitárias, configura-se como uma das mais eficientes medidas de redução de demanda por serviços de atendimento médico, podendo, assim, reduzir, no longo prazo, gastos na área de saúde e melhoria na qualidade do sistema.

Adicionalmente, a ampliação da oferta de escolas para crianças de 4 e 5 anos é de extrema relevância, na medida em que a formação de lares monoparentais e consolidação da mulher no mercado de trabalho configuram-se como um arranjo social atual nas famílias brasileiras. Por fim, iniciar o aprendizado no período correto é condição essencial para o aprimoramento das habilidades cognitivas e melhoria da qualidade da educação.

\section{Referências}

ACEMOGLU, D.; ROBINSON, J. Por que as nações fracassam: as origens do poder, da prosperidade e da pobreza. Rio de Janeiro: Campus, 2012.

ARBEX. M. A. et al. Queima de biomassa e efeitos sobre a saúde. Jornal Brasileiro de Pneumologia, São Paulo, v. 30, n. 2, p. 158-175, 2004.

BARBOSA FILHO, F. H.; PESSÔA, S. Educação, crescimento e distribuição de renda: a experiência Brasileira em perspectiva histórica. In: VELOSO, F. et al. (Org.). Educação básica no Brasil. Rio de Janeiro: Campus, 2009.

BARROS, R. P. et al. A queda recente da desigualdade de renda no Brasil. In: BARROS, R. P.; FOGUEL, M. N.; ULYSSEA, G. (Org.). Desigualdade de renda no Brasil: uma análise da queda recente. Brasília: Ipea, 2007a.

. Acesso ao trabalho e produtividade no Brasil: implicações para crescimento, pobreza e desigualdade. Rio de Janeiro: Ipea, 2004. 
. Consequências e causas imediatas da queda recente da desigualdade de renda Brasileira. Rio de Janeiro: IPEA, jul. 2006b. (Texto para Discussão, 1.201).

. Determinantes da queda na desigualdade de renda no Brasil. In: CASTRO, J. A.; RIBEIRO, J. A. C. Situação Social Brasileira 2007. Brasília, DF: Ipea, 2009 a.

. Determinantes imediatos da queda da desigualdade de renda Brasileira. Rio de Janeiro: IPEA, 2007b. (Texto para discussão, n. 1253).

. Measuring inequality of opportunities in Latin America and the Caribbean. Washington, DC: The World Bank, 2009b.

. Uma Análise das principais causas da queda recente na desigualdade de renda Brasileira. Econômica, Rio de Janeiro, v. 8, n. 1, p. 117-147, 2006 a.

BARROS, R. P.; FRANCO, S.; MEDONÇA, R. A recente queda da desigualdade de renda e o acelerado progresso educacional Brasileiro da última década. Rio de Janeiro: IPEA, set. 2007b. (Texto para discussão, n. 1304).

. Discriminação e segmentação no mercado de trabalho e desigualdade de renda no Brasil. Rio de Janeiro: IPEA, jul. 2007a. (Texto para discussão, n. 1288).

. O papel das transferências públicas na queda recente da desigualdade de renda Brasileira. In: BARROS, R. P.; FOGUEL, M. N.; ULYSSEA, G. (Org.). Desigualdade de renda no Brasil: uma análise da queda recente. Brasília: Ipea, 2007c.

BARROS, R. P.; VEGA, J. R. M.; CHANDUVI, J. S. Measuring inequality of opportunities for children. Washington, DC: The World Bank, 2008.

BARROS, R. P.; VEGA, J. R. M.; SAAVEDRA, J. Measuring progress toward basic opportunities for all. Brazilian Review of Econometrics, Rio de Janeiro, v. 30, n. 2, p. 335-367, nov. 2010.

BOTTON, A. Desejo de status. Rio de Janeiro: Rocco, 2004.

CASTRO, C. M. Investment in education in Brazil: a study of two industrial commuities. Nashville: Vanderbilt University, 1970. (PHD Thesis).

CRISTIA. J. P. et al. Technology and child development: evidence from the one laptop per child program. Germany: Inter-American Development Bank, Feb. 2012. (IDB Working paper, n. 304).

DILL, H. C.; GONÇALVES, F. O. Igualdade de oportunidade no Brasil entre 1999 e 2009: estimação e decomposição através do valor de Shapley. Pesquisa e Planejamento Econômico, Rio de Janeiro, v. 42, n. 2, p. 185-210, 2012.

FERREIRA, F. H. G. et al. Ascensão e queda da desigualdade de renda no Brasil. Econômica, Rio de Janeiro, v. 8, n. 1, p. 147-169, 2006.

FERREIRA, F. H. G. os determinantes da desigualdade de renda no Brasil: luta de classes ou heterogeneidade educacional? In: HENRIQUES, R. (Org.). Desigualdade e pobreza no Brasil. Rio de Janeiro: IPEA, 2000. 
FERREIRA, P. C.; VELOSO, F. O desenvolvimento econômico Brasileiro no pós-guerra. In: VELOSO, F. et al. (Org.). Desenvolvimento econômico: uma perspectiva Brasileira. Rio de Janeiro: Campus, 2013.

. Mobilidade intergeracional de educação no Brasil. Pesquisa e Planejamento Econômico, Rio de Janeiro, v. 33, n. 3, p. 481-513, 2003.

FISHLOW, A. Brazilian size distribution of income. American Economic Review, v. 62, n. 2, p. 391-402, 1972.

FURTADO, C. Um projeto para o Brasil. 3. ed. Rio de Janeiro: Saga, 1968.

HARTUNG. G. C.; PESSÔA, S. Fatores demográficos como determinantes da criminalidade. In: ENCONTRO NACIONAL DE ECONOMIA, 35., 2007, Recife. Anais... Recife: ANPEC, 2007.

HOBBES, T. Leviatã. São Paulo: Martin Claret, 2009.

HOFFMANN, R. As transferências não são a causa principal da redução da desigualdade. Econômica, Rio de Janeiro, v. 7, n. 2, p. 335-341, 2005.

IBGE. Pesquisa Nacional por Amostra de Domicílios (PNAD). Diversos anos. Disponível em: <http://www.ibge.gov.br/home/estatistica/populacao/trabalhoerendimento/pnad2015/ microdados.shtm>. Acesso em: 25 ago. 2017.

KAUFMAN. S. et al. Rural electrification with solar energy as a climate protection strategy. Washington, DC: Renewable Energy Policy Project, 2000. (Research Report, n. 9).

LAM, D.; LEVINSON, D. Declining inequality in schooling in Brazil and its effects on inequality in earnings. Journal of Development Economics, v. 37, n. 1-2, p. 199-225, Nov. 1992.

LAM, D.; SCHOENI, R. Effects of family background on earnings and returns to schooling: evidence from Brazil. Journal of Political Economy, v. 101, n. 4, p. 710-739, Aug. 1993.

LANGONI, C. G. Distribuição de renda e desenvolvimento econômico no Brasil. Rio de Janeiro: FGV, 2005. (Publicado originalmente em 1973).

LEAL, C. I. S.; WERLANG, S. R. C. Retornos em educação no Brasil: 1976/89. Pesquisa e Planejamento Econômico, Rio de Janeiro, v. 21, n. 3, p. 559-574, 1991.

LOCKE, J. Dois tratados sobre o governo. São Paulo: Martins Fontes, 2001.

MEDEIROS. M. As fontes de rendimentos dos ricos no Brasil. Brasília, DF: IPEA, 2004. (Texto para discussão, n. 1014).

. O estudo dos ricos no Brasil. Econômica, Rio de Janeiro, v. 7, n. 1, p. 99-128, 2005. MENEZES-FILHO, N. A. Educação e desigualdade. In: MENEZES-FILHO, N.; LISBOA, M. (Org.). Microeconomia e sociedade no Brasil. Rio de Janeiro: EPGE-FGV, 2001. 
MENEZES-FILHO, N. A.; FERNANDES, R.; PICCHETTI, P. A evolução da distribuição de salários no Brasil: fatos estilizados para as décadas de 80 e 90. In: HENRIQUES, R. (Org.). Desigualdade e pobreza no Brasil. Rio de Janeiro: IPEA, 2000.

MOLINAS, J. R.; BARROS, R. P.; SAAVEDRA, J.; GIUGALE, M. Do our Children have a chance? The 2010 human opportunity report for Latin America and the Caribbean. Washington, DC: The World Bank, 2010.

NOZICK, R. Anarquia, estado e utopia. Rio de Janeiro: Jorge Zahar, 1974.

OSÓRIO. R. G. Berço de ouro: a mobilidade social dos ricos Brasileiros. Econômica, Rio de Janeiro, v. 7, n. 1, p. 129-135, 2005.

RAMOS, L. A desigualdade de rendimentos do trabalho no período pós-real: o papel da escolaridade e do desemprego. Economia Aplicada, São Paulo, v. 11, n. 2, p. 281-301, 2007.

RAWLS, J. Uma teoria da justiça. São Paulo: Martins Fontes, 1971.

REIS, J.; BARROS, R. Desigualdade salarial e distribuição de educação: a evolução das diferenças regionais no Brasil. Pesquisa e Planejamento Econômico, Rio de Janeiro, v. 20, n. 3, p. 415-478, 1990.

. Wage inequality and distribution of education. Journal of Development Economics, v. $\overline{36, \text { n. } 1}$, p. 117-143, July 1991.

REIS, M. C.; RAMOS, L. Escolaridade dos pais, desempenho no mercado de trabalho e desigualdade de rendimentos. Revista Brasileira de Economia, Rio de Janeiro, v. 65, n. 2, p. 177-205, 2011.

ROEMER, J. Equality of opportunity. Cambridge: Harvard University, 1998.

RUTSTEIN, A. Factors associated with trends in infant and child mortality in developing countries during the 1990s. World Health Organization, v. 78, n. 10, p. 1256-1270, 2000.

SCHWARTZMAN, S. As causas da pobreza. Rio de Janeiro: FGV, 2004.

SEN, A. Real national income. Review of Economic Studies, v. 43, n. 1, p. 19-39, 1976.

SOARES, F. V. et al. Programas de transferência de renda no Brasil: impactos sobre a desigualdade. In: BARROS, R. P.; FOGUEL, M. N.; ULYSSEA, G. (Org.). Desigualdade de renda no Brasil: uma análise da queda recente. Brasília: Ipea, 2007.

SOARES, S. S. D. Análise do bem-estar e decomposição por fatores da queda na desigualdade entre 1995 e 2004. Econômica, Rio de Janeiro, v. 8, n. 1, p. 83-115, 2006.

VELOSO, F. 15 Anos de avanço na educação no Brasil: onde estamos? In: VELOSO, F.; PESSÔA, S.; HENRIQUES, R.; GIAMBIAGI, F. (Org.). Educação básica no Brasil. Rio de Janeiro: Campus, 2009.

VILLELA, A. O desenvolvimento econômico no Brasil pré-1945. In: VELOSO, F. et al. (Org.). Desenvolvimento econômico: uma perspectiva Brasileira. Rio de Janeiro: Campus, 2013. 
WORLD BANK. World development report 2006: equity and development. Washington, DC: The World Bank, 2006.

\section{Apêndice A - Índice de Oportunidade Humana (IOH) a partir da Taxa de Cobertura (c) e do Indice de Desigualdade (D)}

A maximização do IOH é feita através da combinação da expansão da taxa de cobertura (C) com uma alocação mais eficiente da igualdade de oportunidades (D). Em Barros et al. (2009), o IOH é a composição de uma média simples de duas dimensões (educação e habitação/infraestrutura). Por sua vez, a dimensão educação é subproduto de outras duas, enquanto que a dimensão habitação/infraestrutura é a média simples de outras três. ${ }^{13}$

Analiticamente, pode-se considerar como cada circunstância contribui para a desigualdade total de oportunidade. Assim, calcula-se uma medida específica relacionada com uma determinada circunstância $x$, isto é, um índice de desigualdade $D$ condicionado a variação de uma única circunstância, mantendo as demais constantes, ${ }^{14}$ nos seguintes termos:

$$
\begin{aligned}
& p(x)=P[I=1 \mid x]=E[I \mid x] \\
& q\left(x_{k}\right)=\int p(x) d F\left(x_{-k}\right)
\end{aligned}
$$

Se a circunstância para a criança for aquela associada à área de residência, por exemplo, estima-se para cada observação sua probabilidade de acesso. Assim, tem-se uma probabilidade média $q$ para a área urbana e uma para a área rural.

Considere, assim, uma população de $N$ indivíduos divididos entre $m$ grupos de circunstâncias disjuntos. Seja também a circunstância j com $M_{j}$ o número de sujeitos em um grupo com acesso à oportunidade em análise (isto é, para $I=1$ )

13 Neste caso, para um IOH de 0,6, significa que $60 \%$ das oportunidades proporcionadas pelas condições de educação e/ou habitação são igualmente distribuídas entre a população de crianças de 0 a 16 anos de idade.

14 Um perfil de desigualdade de oportunidade pode ser definido pelo tamanho relativo de cada $D$ para uma circunstância específica. Barros et al. (2009), ao considerar a circunstância educação dos pais, estimam que, para o Brasil, 11,7\% das oportunidades educacionais disponíveis precisam ser realocadas para eliminar as diferenças na dimensão do término da $6^{\mathrm{a}}$ série na idade correta entre diferentes grupos. Quando considerada apenas a dimensão da área residente (região urbana), apenas $2,7 \%$ das oportunidades educacionais disponíveis precisam ser realocadas, a fim de eliminar as diferenças na conclusão da $6^{a}$ série na idade correta. É também possível observar que a desigualdade de oportunidades resultante exclusivamente da diferença na educação dos pais é maior do que a desigualdade resultante de qualquer outra circunstância analisada (gênero, raça, renda familiar per capita, presença dos pais e número de irmãos). 
e $N_{j}$ o número total de indivíduos nesse grupo. Denotando $\bar{M}_{j}$ o número de indivíduos que têm acesso à oportunidade avaliada para os distintos grupos, tem-se que:

$$
\bar{M}_{j}=\bar{p} N_{j}
$$

A diferença de acesso à oportunidade para todos os grupos de circunstâncias e o número mínimo de oportunidades que precisam ser reorganizadas para alcançar a igualdade de acesso em todos os grupos são dados, respectivamente, por $\left|M_{j}-\bar{M}_{j}\right|$ e $\frac{1}{2} \sum_{j=1}^{m}\left|M_{j}-\bar{M}_{j}\right|$. Segue-se, então, que o percentual do número total de oportunidades disponíveis é dado por:

$$
M=\sum_{j=1}^{m} M_{j}=\bar{p} N
$$

Dessa forma, a quantidade de acesso que precisa ser alocada para garantir igualdade de acesso considerando todos os grupos de circunstâncias pode ser expressa por:

$$
\begin{gathered}
D=\frac{1}{2 \bar{p} N} \sum_{j=1}^{m}\left|M_{j}-\bar{M}_{j}\right| \\
D=\frac{1}{2 \bar{p} N} \sum_{j=1}^{m}\left|M_{j}-\bar{M}_{j}\right|=\frac{1}{2-} \frac{N_{j}}{N} \sum_{j=1}^{m} \frac{\left|M_{j}-\bar{M}_{j}\right|}{N_{j}}=\frac{1}{2-} \sum_{j=1}^{m} \beta_{j}\left|p_{j}-\bar{p}_{j}\right|
\end{gathered}
$$

Seja também $\gamma_{j}$ a proporção das possibilidades globais disponíveis que estão atualmente atribuídos ao grupo j. Assim, $\gamma_{j}=\frac{M_{j}}{M}$ e podemos reescrever $D$ como:

$$
D=\frac{1}{2 \bar{p} N} \sum_{j=1}^{m}\left|M_{j}-\bar{M}_{j}\right|=\frac{1}{2 M} \sum_{j=1}^{m}\left|M_{j}-\bar{p} N_{j}\right|=\frac{1}{2} \sum_{j=1}^{m}\left|\gamma_{j}-\beta_{j}\right|
$$


Nesse caso, $D$ pode ser interpretado como a distância entre a distribuição das oportunidades $\left\{\gamma_{j}\right\}$ e a distribuição da população $\left\{\beta_{j}\right\}$ em todos os grupos de circunstâncias.

Resta ainda a construção de uma medida distributiva que seja relacionada à desigualdade de oportunidade. Assim, $\bar{p}=\frac{M}{N}$ seria a percentagem do número total de oportunidades necessárias para o acesso universal que estão realmente disponíveis. Neste caso, $p$ pode entendido como uma medida do estoque de oportunidades disponíveis, ao mesmo tempo em que é insensível à alocação das oportunidades. Pode-se, todavia, redimensionar o numerador em questão alocando aqueles de acordo com o princípio da igualdade de oportunidades. Portanto, sendo $r$ as oportunidades disponíveis alocadas, tem-se que:

$$
I O H=\frac{r}{N}
$$

Como $(1-D)$ corresponde à percentagem de oportunidades disponíveis que foram igualmente distribuídas e, portanto, $M(1-D)$ seu total de acordo com o princípio da igualdade de oportunidades, o $I O H=M(1-D)$ será assim definido:

$$
I O H=\frac{r}{N}=\frac{M}{N}(1-D)=\bar{p}(1-D)
$$

Recebido em: 20/12/2013. Aceito em: 04/10/2016. 